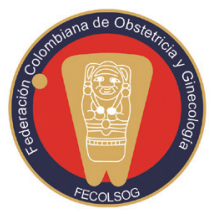

\title{
ÓBITO FETAL: HALLAZGOS DE PATOLOGÍA EN UNA INSTITUCIÓN DE ALTA COMPLEJIDAD. CARTAGENA, COLOMBIA, 2010-2011
}

\author{
Stillbirth: Pathologic findlings a tertiary care \\ hospital. Cartagena, Colombia, 2010-2011
}

Edgar E. Rivas-Perdomo, $M^{1}{ }^{1}$; Doris Vásquez-Deulofeutt, Enf. ${ }^{2}$

Recibido: junio 27/12 - Aceptado: diciembre 13/12

\section{RESUMEN}

Introducción: conocer las características epidemiológicas del óbito fetal y describir los hallazgos más frecuentes en los estudios de patología de mortinatos que se atienden en la Clínica Universitaria San Juan de Dios.

Materiales y métodos: se presenta una serie de casos de todos los mortinatos atendidos durante el periodo comprendido entre junio de 2010-mayo de 2011, en una institución de alta complejidad en Cartagena, Colombia. Se establecieron tres grupos de edad gestacional al momento de la muerte fetal: 22 a $29+6,30-36+6$ y 37-42 semanas de gestación. Se consideraron variables demográficas, antecedentes maternos, resultado del embarazo y los hallazgos de patología en el feto, la placenta y el cordón. Los resultados se presentan mediante estadística descriptiva.

Resultados: la edad promedio fue de 29,1 años SD: 6,1. El 72,2\% de los casos se presentó en pacientes entre los 20 a 34 años. La patología placentaria se

Especialista en Ginecología y Obstetricia, Universidad de Cartagena, Colombia. Candidato a Maestría en Epidemiología Clínica, Universidad de La Frontera, Chile. Clínica Universitaria San Juan de Dios. Cartagena, Colombia. erivasperdomo@gmail.com

Enfermera, Universidad de Cartagena. Coordinadora Servicio Ginecología y Obstetricia, Clínica Universitaria San Juan de Dios. Cartagena, Colombia. identificó como causa primaria de óbito fetal en el 25\% de los casos. Por otra parte, en el 22,22\% de los casos no se demostró la causa primaria de óbito fetal.

Conclusiones: el estudio de patología aportó información para definir la causa de muerte en el 77\% de los casos. Este examen aporta información útil para la toma de decisiones a nivel clínico y en salud pública.

Palabras clave: óbito fetal, muerte fetal intrauterina, patología.

\section{ABSTRACT}

Objective: Ascertaining stillbirth's epidemiological characteristics and describing the most frequent findings in studies of stillbirths attended at the San Juan de Dios teaching hospital.

Materials and methods: A case series was compiled of all stillbirths attended from June 2010 to May 2011 at a high complexity institution in Cartagena, Colombia. Three groups of gestational age at the moment of fetal death were established: 22 to $29+6,30$ to $36+6$ and 37 to 42 weeks' gestation. Demographic variables, maternal background, the result of pregnancy and findings regarding disease in the fetus, placenta and umbilical cord were all considered. Descriptive statistics were used to describe the sample. 
Results: Average age was 29.1 (6.1 SD); 72.2\% of the cases occurred in 20- to 34-year-old patients. Placental disease was identified as the primary cause of stillbirth in $25 \%$ of the cases; nevertheless, the primary cause of stillbirth could not be demonstrated in $22.22 \%$ of them.

Conclusions: Studying the disease provided information for defining the cause of death in $77 \%$ of the cases. This exam supplied useful information for decision-making at clinical level and in public health. Key words: Stillbirth, intrauterine fetal death, disease.

\section{INTRODUCCIÓN}

La muerte fetal se define como aquella que ocurre después de las 20 semanas de gestación o con un peso fetal $>500$ g cuando la edad gestacional no es segura. Es uno de los resultados adversos más comunes en el mundo. En Estados Unidos ocurre en el 7 por 1000 de todos los nacimientos. En algunos países africanos solamente a causa de la malaria se tienen tasas de mortalidad fetal de 40 por 1000 nacimientos (1). En Chile este problema tiene una frecuencia entre 7 a 10 por 1000 nacidos vivos (2). En Venezuela se reporta una tasa de 16,52 muertes fetales tardías por cada 1000 nacidos vivos (3). En Estados Unidos, las tasas de mortalidad fetal han declinado solo en $17 \%$ entre 1985 y 2001, de 7,8 a 6,5 muertes por 1000 nacimientos, comparado con una reducción en un 35\% de la mortalidad infantil en ese periodo de tiempo (3).

Para diseñar acciones eficaces se necesita: 1) identificar los factores de riesgo más asociados con su ocurrencia; 2) conocer sus principales causas, 3) evaluar su comportamiento epidemiológico, 4) reconocer que los daños a la salud perinatal tienen una vinculación estrecha con los factores de riesgo y las patologías que afectan a la madre desde la etapa preconcepcional, durante el embarazo y el parto (5).

Es escasa la literatura colombiana a este respecto, dado que en la actualidad la autopsia fetal no es práctica frecuente en nuestras instituciones hospitalarias. Conocer esta información podría ser útil para definir posibles intervenciones dirigidas a reducir el impacto de los factores de riesgo o las entidades asociadas a la muerte fetal. Se pretende determinar las características epidemiológicas de la entidad y determinar los hallazgos más frecuentes en los estudios de patología de mortinatos que se atienden en la Clínica Universitaria San Juan de Dios en Cartagena, para brindar información a los tomadores de decisiones sobre problemas que se deben intervenir en este campo.

\section{MATERIALES Y MÉTODOS}

Se realizó un estudio observacional descriptivo tipo serie de casos. Se incluyeron todos los casos de mortinatos que fueron atendidos en la Clínica Universitaria San Juan de Dios (Cartagena, Colombia) durante el tiempo comprendido entre el 1 de junio de 2010 hasta el 31 de mayo de 2011. Esta es una institución de tercer nivel de complejidad que atiende pacientes pertenecientes al régimen de aseguramiento contributivo privado. Se excluyeron los sujetos que tenían reclamaciones médico-legales y aquellos cuyos familiares no autorizaron el procedimiento. Se realizó muestreo consecutivo.

Procedimiento. Se revisaron los expedientes clínicos de todas las pacientes con diagnóstico de muerte fetal intrauterina con 22 o más semanas de gestación que ingresaron a la clínica durante el tiempo de estudio. Se contó con la aprobación del comité de ética de la institución, y se solicitó la firma del consentimiento de los padres para la realización del procedimiento y la inclusión de los datos en el estudio.

La edad gestacional se determinó por la historia menstrual cuando fue confiable, o por biometría fetal ultrasonográfica previa al ingreso. De acuerdo con la edad gestacional en que ocurrió la muerte fetal se establecieron tres grupos: 2229+6, 30-36+6 y 37-42 semanas de gestación. La clasificación etiológica fue posible luego de analizar en conjunto los antecedentes clínicos y de laboratorio maternos con los hallazgos anatomopatológicos feto-placentarios. El estudio histológico feto-placentario fue realizado por el servicio de 
Patología contratado por esta institución. Los casos sin antecedentes maternos y con feto en estado de maceración avanzada o placenta con alteraciones involutivas (cambios secundarios a muerte fetal) fueron catalogados como no clasificables. Se determinó la edad, la procedencia, el estado civil, el tipo de seguridad social y el número de partos atendidos en la institución; la paridad, las pérdidas fetales previas, los antecedentes médicos y la vía del parto; así como el sexo, el peso y la talla, y hallazgos de patología en el feto, la placenta y el cordón. Para la determinación de las causas primarias de muerte fetal se siguió la clasificación propuesta por Ovalle en 2005 (2). Para el análisis se creó una base de datos utilizando un software Epi-Info 2004, versión .2, y se usó el programa Excel Microsoft. Se describen las variables continuas por medio de medidas de tendencia central y de dispersión; las variables categóricas se presentan por medio de proporciones.

\section{RESULTADOS}

Se atendieron 4254 nacimientos y ocurrieron 39 muertes fetales $(9,16 / 1000$ nacidos vivos) de los cuales 36 cumplieron con los criterios de inclusión, que corresponden al 92,30\% de las muertes fetales. Se excluyeron 3 casos: 2 por reclamaciones médicolegales y un caso en que los familiares no autorizaron el procedimiento. Respecto a las características de la población estudiada la edad promedio fue de 29,1 años $( \pm 6,1)$. Se destaca la menor frecuencia del evento en el grupo de adolescentes. Se encontró que el 58,3\% de la gestantes tenía menos de 3 embarazos anteriores y el 41,7\% tenían 3 o más embarazos previos. La mayoría de las pacientes $(83,3 \%)$ no había tenido óbitos previos, mientras que el 13,9\% había tenido una pérdida anterior, y el 2,8\% había tenido 2 pérdidas. El peso fetal promedio fue de $1613 \mathrm{~g}(+$ 1021), rango: 500,0-3865; q25-75: 1450-2009. En cuanto a la talla la media fue $39,75( \pm 9,50)$, rango: 15,0 -50,0; q25-75: 45-47,5. El 11,11\% de los fetos tenían malformaciones estructurales, principalmente defectos del tubo neural. La vía de elección para el parto fue la vaginal; los casos en que se escogió la cesárea respondieron principalmente al hecho de antecedentes de este procedimiento (tabla 1).

\begin{tabular}{|c|c|c|}
\hline $\begin{array}{r}\text { Caracter } \\
\text { que present } \\
\text { Sar }\end{array}$ & $\begin{array}{l}\text { a poblac } 1 . \\
\text { al. Clínic } \\
\text { Dios, Car }\end{array}$ & $\begin{array}{l}\text { gestante } \\
\text { niversitaria } \\
\text { ha }\end{array}$ \\
\hline & $=36$ & \\
\hline Edad (años) & Número & Porcentaje \\
\hline $16-19$ & 2 & 5,6 \\
\hline $20-34$ & 26 & 72,2 \\
\hline$\geq 35$ & 8 & 22,2 \\
\hline
\end{tabular}

Estado civil

\begin{tabular}{|l|l|l|}
\hline 1. Soltera & 10 & 27,80 \\
\hline 2. Unión estable & 26 & 72,20 \\
\hline
\end{tabular}

Seguridad social

\begin{tabular}{|l|c|c|}
\hline 2. Subsidiado & 8 & 22,2 \\
\hline 3. Contributivo & 28 & 77,80 \\
\hline
\end{tabular}

Procedencia

\begin{tabular}{|l|c|c|}
\hline 1. Urbana & 35 & 97,20 \\
\hline 2. Rural & 1 & 2,80 \\
\hline
\end{tabular}

Edad gestacional

\begin{tabular}{|l|l|l|}
\hline EG (semanas) & & \\
\hline$<30$ S & 14 & 38,90 \\
\hline $30-36$ & 12 & 33,33 \\
\hline$>37$ & 10 & 27,77 \\
\hline
\end{tabular}

Producto

\begin{tabular}{|l|c|c|}
\hline$<750$ & 10 & 27,77 \\
\hline $750-1499$ & 8 & 22,22 \\
\hline $1500-2499$ & 10 & 27,77 \\
\hline $2500->4000$ & 8 & 22,22 \\
\hline
\end{tabular}

\section{Sexo}

Sexo. 1: masculino

Sexo. 2: femenino

\begin{tabular}{|l|r|}
\hline 15 & 41,7 \\
\hline 21 & 58,3 \\
\hline
\end{tabular}

Malformaciones fetales

\begin{tabular}{|l|c|c|}
\hline 1. Sí & 4 & 11,11 \\
\hline 2. No & 32 & 88,89 \\
\hline
\end{tabular}

Vía del parto

\begin{tabular}{|l|l|l|}
\hline 1. Vaginal & 21 & 58,30 \\
\hline 2. Cesárea & 15 & 41,70 \\
\hline
\end{tabular}

Fuente: archivos Clínica Universitaria San Juan de Dios, Cartagena. 
Respecto a la causa de muerte se encontró que la patología placentaria fue la causa primaria de óbito fetal, seguida de las enfermedades maternas. En nuestra serie no se pudo demostrar la causa primaria de muerte fetal en el 22,22\% de los casos. En cuanto a los cuatro casos de muerte fetal a causa de enfermedad materna, tres fueron por trastorno hipertensivo del embarazo, lo cual siempre se ha establecido como riesgo para el resultado adverso (tabla 2). Las causas de patología placentaria se describen en la tabla 3.

\section{DISCUSIÓN}

En nuestro estudio se estableció una tasa de 9,16/1000 nacidos vivos, la cual es más elevada que la reportada por otros autores $(1,2)$. En los países industrializados se estimó para el año 2000 una tasa de 4,2-6,8/1000 nacidos vivos, muy inferior a la encontrada en los países pobres, que representa 20-32/1000 nacidos vivos (6). La tasa de óbito fetal en Estados Unidos después de declinar dramáticamente de 20 por cada 1000 nacidos vivos en 1950, se ha mantenido constante en 6,3/1000 nacidos vivos a partir del 2003.
Existen diferencias en cuanto a los resultados reportados por diferentes autores respecto a la relación de la edad materna con la muerte fetal (3, $6,7)$. Se ha considerado que la edad materna de 35 años otorga un mayor riesgo; sin embargo, Milla en su serie reporta que el 23,4\% de las pacientes tenía más de 35 años (8), lo cual es similar a lo reportado en nuestra serie.

En la literatura hay controversia sobre el papel de la multiparidad como factor de riesgo de muerte fetal. Respecto a la pérdida fetal previa nuestros hallazgos no son concordantes con lo reportado en la literatura acerca de que los óbitos previos incrementan el riesgo de pérdidas fetales en los embarazos subsiguientes (9), pero se corresponde con Sharma en el 2007 quien encontró que el resultado de embarazos que siguen a una pérdida fetal en la primera gestación era similar en aquellas mujeres que tuvieron parto de un recién nacido vivo en el primer embarazo (10). Las muertes fetales recurrentes son menos entendidas y se estima que afectan del 0,5-3\% de las mujeres en edad reproductiva (11).

Se ha considerado que la muerte fetal es rara en el segundo trimestre (12), y aunque en nuestra

\begin{tabular}{|c|c|c|c|c|c|}
\hline & $\begin{array}{l}\text { Tabla 2. Causas } \\
\text { Clínica Universitaria }\end{array}$ & $\begin{array}{l}\text { imarias de } \\
\text { in Juan de I }\end{array}$ & $\begin{array}{l}\text { ito fetal. } \\
\text { os, Cartagen }\end{array}$ & & \\
\hline \multirow[t]{2}{*}{ Causas primarias } & \multirow[t]{2}{*}{ Subgrupos } & \multicolumn{3}{|c|}{ Edades (años) } & \multirow[t]{2}{*}{ Totales } \\
\hline & & 16-19 & $20-34$ & $\geq 35$ & \\
\hline \multirow[t]{4}{*}{ Hipoxia fetal extrínseca } & Patología placentaria & 1 & 7 & 1 & $9(25,00 \%)$ \\
\hline & Cordón umbilical & 0 & 0 & 1 & $1(2,77 \%)$ \\
\hline & Enfermedades maternas & 1 & 1 & 2 & $4(11,11 \%)$ \\
\hline & No determinadas & 0 & 7 & 1 & $8(22,22 \%)$ \\
\hline Infecciones ascendentes & & 0 & 7 & 3 & $10(27,77 \%)$ \\
\hline Anomalías congénitas & & 1 & 1 & 0 & $2(5,55 \%)$ \\
\hline Accidentes labor de parto & & 1 & 0 & 0 & $1(2,77 \%)$ \\
\hline No identificables & & 0 & 1 & 0 & $1(2,77 \%)$ \\
\hline Totales & & $4(11,11 \%)$ & $24(66,66 \%)$ & $8(22,22 \%)$ & 36 \\
\hline
\end{tabular}

Fuente: archivos Clínica Universitaria San Juan de Dios, Cartagena. 


\begin{tabular}{|c|c|}
\hline \multicolumn{2}{|c|}{$\begin{array}{c}\text { Tabla } 3 . \\
\text { Hallazgos de patología en placenta } \\
\text { y cordón de óbito fetal. } \\
\text { Clínica Universitaria San Juan }\end{array}$} \\
\hline Placenta & $\mathbf{N}$ \\
\hline Corioamnionitis & 13 \\
\hline Depósito de fibrina intervellosa & 9 \\
\hline Maduración irregular de cotiledones & 7 \\
\hline Infartos placentarios & 6 \\
\hline Nudos sincitiales & 3 \\
\hline Deciduitis & 3 \\
\hline Hematomas retroplacentarios & 2 \\
\hline Microcalcificaciones & 2 \\
\hline Funisitis & 2 \\
\hline Fenómenos trombóticos & 2 \\
\hline Hipoplasia de vellosidades & 2 \\
\hline Cambios por retención & 1 \\
\hline Corangiosis focal & 1 \\
\hline Hemorragia intervellosa & 1 \\
\hline Trombos intervellosos & 1 \\
\hline Abruptio & 1 \\
\hline Congestión vascular generalizada & 1 \\
\hline Vellitis & 1 \\
\hline Endarteritis obliterante & 1 \\
\hline \multicolumn{2}{|l|}{ Cordón umbilical } \\
\hline Congestión vascular & 2 \\
\hline Nudo verdadero apretado & 1 \\
\hline Onfalitis & 4 \\
\hline Trombosis venosa & 1 \\
\hline
\end{tabular}

Fuente: archivos Clínica Universitaria San Juan de Dios, Cartagena.

serie no se dividieron las edades gestacionales por trimestres, sino considerando la viabilidad fetal, no se observa marcada diferencia en los mismos grupos.

Acerca de la ausencia de causa específica de muerte fetal hay que recordar que en algunos casos es difícil determinar su etiología a pesar de investigarla, y que más de una condición puede contribuir a la muerte fetal en un caso particular $(4,13)$. La mayoría de las causas de muerte fetal diferentes a anomalías congénitas e infecciones está relacionada con la alteración de la placentación y con la función placentaria hasta en un 65\% (13). Hom, en su serie, no la pudo establecer en el 15,2\% de los casos (14); según Sepúlveda en el 2004, el porcentaje de muerte fetal inexplicada osciló entre el 21 al $50 \%$ de los casos (15). El estudio de las causas por medio del diagnóstico anatomo-patológico y la identificación de los factores maternos que se asocian a esta entidad cobra importancia a fin de buscar factores en los que se pueda trabajar para evitarlos (8); la tasa de "muerte fetal no explicada" difiere entre los diferentes países dependiendo de los recursos disponibles y del interés que se tenga. En los países con pocos recursos con frecuencia es más difícil de determinar (16).

Por otro lado, la autopsia podría revelar la presencia de anomalías congénitas o evidencia de hipoxia ya sea aguda o crónica. Además, la autopsia del feto y el examen patológico de la placenta podrían demostrar patologías asociadas con la muerte fetal que incluyen dismadurez vellositaria, patología uteroplacentaria, infecciones con inflamación consistente, patología del cordón, infartos placentarios y raramente tumores placentarios (1). Respecto a la alta frecuencia de corioamnionitis encontrada por nosotros, Lahra reporta en su serie una incidencia de corioamnionitis histológica del 36,9\% (17). Estos datos se corresponden con los reportados por Ovalle et al. (2). Por ello, y de acuerdo con el mismo autor, la recomendación de pesquisa y tratamiento de las infecciones cérvico-vaginales y urinarias en la población de riesgo debe hacerse desde el inicio del segundo trimestre del embarazo (2). No obstante, es preciso tener en cuenta que por varias razones no es muy clara la relación entre infección materna y muerte fetal. Primero, es difícil conocer la vía exacta por la cual ocurre, y la presencia del organismo en la placenta o el feto no prueba la causalidad $(18,19)$. La poca frecuencia de óbitos relacionados con patología materna podría estar relacionada con la tendencia a interrumpir el embarazo en trastornos severos o de difícil control. En estos casos, el parto pretérmino reduce el riesgo de muerte fetal (20). 


\section{CONCLUSIÓN}

El estudio de patología aportó información para definir la causa de la muerte en el 77\% de los casos. Este examen aporta información útil para la toma de decisiones a nivel clínico y en salud pública.

\section{REFERENCIAS}

1. Rowland A, Goodnight WH. Fetal loss: Addressing the evaluation and supporting the emotional needs of parents. J Midwifery Womens Health 2009;54:241-8.

2. Ovalle A, Kakarieka E, Ángel Correa A, Vial MT, Aspillaga C. Estudio anátomo-clínico de las causas de muerte fetal. Rev Chil Obstet Ginecol 2005;70:303-12.

3. Rojas E, Salas K, Oviedo G, Plenzyk G. Incidencia y factores de riesgo asociados al óbito fetal en 2 hospitales venezolanos. Rev Chil Obstet Ginecol 2006;71:26-30.

4. Silver RM, Varner MW, Reddy U, Goldenberg R, Pinar $\mathrm{H}$, Conway D, et al. Work-up of stillbirth: a review of the evidence. Am J Obstet Gynecol 2007; 196:433-44.

5. Velaszo-Murillo V, Palomares-Trejo A, NavarreteHernández E. Causalidad y tendencia de la mortalidad perinatal hospitalaria en el IMSS, 1998-2002. Cir Ciruj 2003;71:304-13.

6. Yakoob MY, Lawn JE, Darmstadt GL, Bhutta ZA. Stillbirths: epidemiology, evidence, and priorities for action. Semin Perinatol 2010;34:387-94.

7. Reddy UM, Ko CW, Willinger M. Maternal age and the risk of stillbirth throughout pregnancy in the United States. Am J Obstet Gynecol 2006; 195:764-70.

8. Milla LM, Saravia N, Salviz M, Rojas J. Muerte fetal en el Hospital Nacional Arzobispo Loayza durante el periodo agosto 2003-noviembre 2004. Rev Med Hered 2005;16:260-5.
9. Weeks JW. Antepartum testing for women with previous stillbirth. Semin Perinatol 2008;32:301-6.

10. Sharma P, Salihu H, Kirby R. Stillbirth recurrence in a population of relatively low-risk mothers. Paediatr Perinat Epidemiol 2007:21:S24-30.

11. Salihu HM. Fetal death repetition: the event memory Hypothesis. Medical Hypotheses 2008;70:567-71.

12. Michels TC, Tiu A. Second Trimester Pregnancy Loss, Am Fam Physician 2007;76:1341-6.

13. Molina S, Alfonso A. Muerte fetal anteparto. Univ Méd 2010;51:59-73.

14. Horn L, Langner A, Stiehl P, Wittekind CH, Faber R. Identification of the causes of intrauterine death during 310 consecutive autopsies. Eur J Obstet Gynecol Reprod Biol 2004;113:134-8.

15. Sepúlveda J, Quintero E. Muerte fetal inexplicada. Rev Colomb Obstet Ginecol 2004;55:300-7.

16. Gravett MG, Rubens CE, Nunes TM. Gapps Review Group. Global report on preterm birth and stillbirth (2 of 7): discovery science. BMC Pregnancy and Childbirth 2010;10:S2.

17. Lahra MM, Gordon A, Jeffery HE. Chorioamnionitis and fetal response in stillbirth. Am J Obstet Gynecol 2007;196:229.

18. McClure EM, Goldenberg RL. Infection and stillbirth. Sem Fetal Neonatal Med 2009;14:182-9.

19. Goldenberg RL, McClure EM, Saleem S, Reddyajbt UM. Infection-related stillbirths. Lancet 2010;375:1482-90.

20. Aagaard-Tillery KM, Holmgren C, Lacoursiere DY, Houssain S, Bloebaum L, Satterfield R, et al. Factors associated with nonanomalous stillbirths: The Utah Stillbirth Database 1992-2002. Am J Obstet Gynecol 2006;194:849-54.

Conflicto de intereses: ninguno declarado.

Este trabajo fue presentado en el XXVIII Congreso Colombiano de Obstetricia y Ginecología, Cartagena, Colombia, 2012. 